\title{
Radiographic and chest CT imaging presentation and follow-up of COVID-19 pneumonia: a multicenter experience from an endemic area
}

\author{
Federica Vernuccio ${ }^{1,2,3,4}$ (D) Dario Giambelluca ${ }^{5} \cdot$ Roberto Cannella $^{4} \cdot$ Francesco Paolo Lombardo ${ }^{6} \cdot$ Fabio Panzuto $^{7}$. \\ Massimo Midiri $^{4} \cdot$ Paolo Cabassa $^{5}$
}

Received: 23 May 2020 / Accepted: 28 June 2020 / Published online: 11 July 2020

(C) American Society of Emergency Radiology 2020

\begin{abstract}
COVID-19 has infected more than 2 million people in the world in less than 5 months outbreak. Chest imaging is recommended for triage of suspected cases of COVID-19 with moderate-severe clinical features and high pre-test probability of disease, and may help for patient follow-up and to identify patients at higher risk of disease worsening. This pictorial essay illustrates typical and uncommon imaging findings of COVID-19 pneumonia and the role of imaging for patient management.
\end{abstract}

Keywords CT $\cdot$ SARS-CoV-2 $\cdot$ Coronavirus disease 2019 (COVID-19) $\cdot$ Pulmonary embolism $\cdot$ Coronavirus infections

\section{Background}

Coronavirus disease-19 (COVID-19) —also known as 2019 novel coronavirus - has infected more than 2 million people in the world in less than 5 months, with almost 160 thousands deaths [1]. Patients with COVID-19 can progress from asymptomatic or mild disease to hypoxemic respiratory failure or multisystem organ failure, requiring intubation and intensive care management [2].

Federica Vernuccio

federica.vernuccio@unipa.it; federicavernuccio@gmail.com

Dario Giambelluca

dario.giambelluca@asst-franciacorta.it

Roberto Cannella

rob.cannella89@gmail.com

Francesco Paolo Lombardo

francescopaolo.lombardo87@gmail.com

Fabio Panzuto

f.panzuto88@gmail.com

Massimo Midiri

massimo.midiri@unipa.it

Paolo Cabassa

paolo.cabassa@asst-franciacorta.it
The lung is the predominant disease site in COVID-19 infection and may lead to the so-called severe acute respiratory syndrome coronavirus 2 (SARS-CoV-2) infection [2]. COVID-19 infects human cells through its spike surface glycoprotein that binds to the receptor-binding domains of the angiotensin converting enzyme 2 (ACE2), which is abundant in epithelial cells of the tongue and type II alveolar cells [3, 4]. After binding to the ciliated cells in the alveoli, the virion releases RNA into the cell, initiating replication of the virus which further disseminates

1 Department of Health Promotion, Mother and Child Care, Internal Medicine and Medical Specialties, University Hospital of Palermo, Via del Vespro 129, 90127 Palermo, Italy

2 University Paris Diderot, Sorbonne Paris Cité, Paris, France

3 I.R.C.C.S. Centro Neurolesi Bonino Pulejo, Contrada Casazza, SS113, 98124 Messina, Italy

4 Section of Radiology - BiND, University Hospital "Paolo Giaccone", Via del Vespro 129, 90127 Palermo, Italy

5 Department of Radiology, ASST Franciacorta, Azienda Ospedaliera M. Mellini, Viale Mazzini 4, 25032 Chiari, BS, Italy

6 Department of Radiology, ASST Valtellina ed Alto Lario Ospedale "E. Morelli", Via Zubiani 33, 23035 Sondalo, SO, Italy

7 Department of Radiology, ASST Lariana Ospedale "S. Anna”, Via Ravona, 20, 22042 San Fermo della Battaglia, CO, Italy 
to infect other cells. As a consequence, the infected cells likely stop clearing the airways with a consequent progressive accumulation of debris and fluids in the lungs [5-7]. The first non-severe stage of lung involvement by COVID-19, with only mild symptoms, may be attributed to the direct viral damage and innate immune response. The second severe stage, with progressive hypoxemic respiratory failure, may be attributed to tissue damage by specific immune response (the so-called cytokine release syndrome) [8]. Imaging allows to identify and grade the extent of the COVID-19 disease [2]. In this essay, we collected the imaging and clinical experience from multiple Italian centers involved in the COVID-19 outbreak, including two nonacademic hospitals and one academic hospital in Lombardy (the most affected Italian region) that acquired each about 30-60 radiographies and CT scans per day in COVID-19 patients and one academic hospital in Sicily (one of the least affected Italian region).

The aim of this pictorial essay is to illustrate the imaging presentation of COVID-19 pneumonia, including identification of typical and uncommon imaging findings at diagnosis and follow-up and the role of imaging features for patient management.

\section{Imaging presentation and follow-up of COVID-19 pneumonia}

\section{Imaging modalities}

Imaging assessment of COVID-19 pneumonia has been performed with different modalities, including ultrasound (US), chest radiographs (CXR), and computed tomography (CT). The choice of imaging technique depends on equipment availability and clinical context, as well as national/ regional needs and recommendations [2,9]. Lung ultrasound has been proposed in suspected COVID-19 patients as a potential triage and diagnostic tool in tents or other areas outside hospitals where other imaging modalities are not available because it allows to rapidly triage patients through
Fig. 1 A 56-year-old man with fever, cough, and positive for COVID-19. Chest radiograph (a, b) shows no significant lung abnormalities. Coronal (c) and sagittal (d) CT images demonstrate subpleural consolidations and ground-glass opacities in the right lower lobe (arrows)

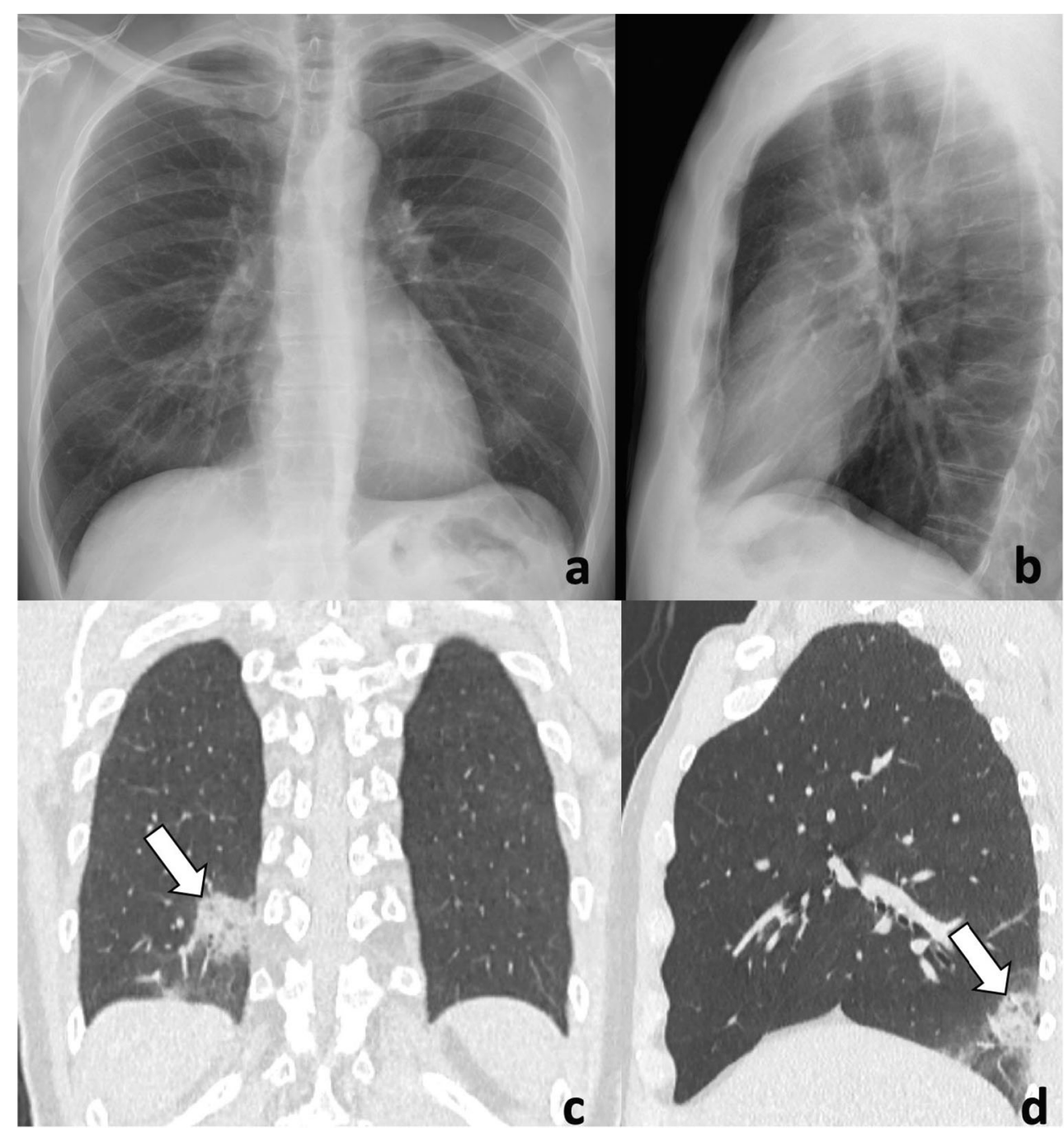




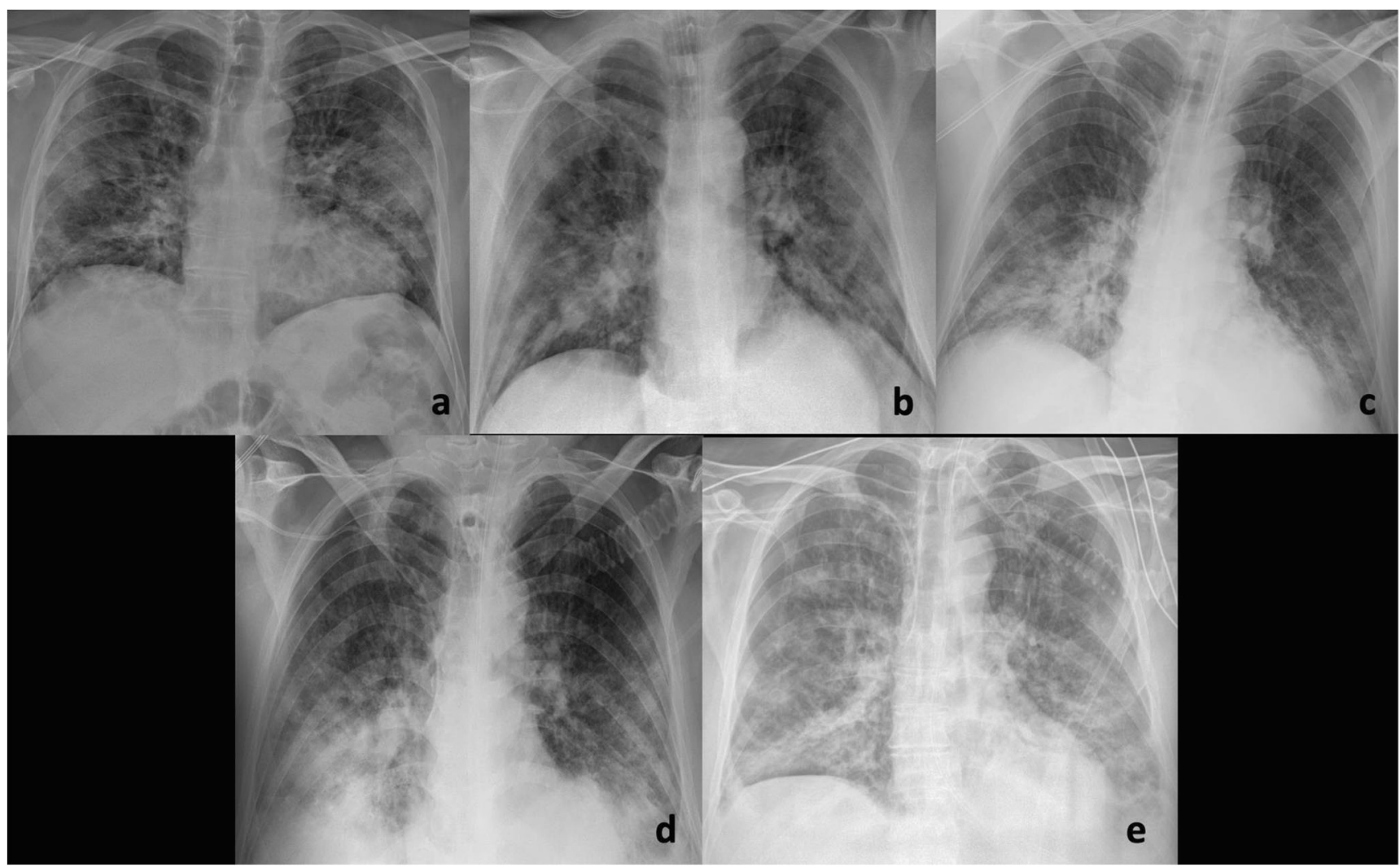

Fig. 2 A 72-year-old man with fever and cough for 7 days and COVID19 pneumonia in chest imaging. Serial chest radiographs performed at admission (a), $12 \mathrm{~h}$ after tracheal intubation in ICU (b), 5 days (c), 10 days (d), and 12-days (e) of ICU demonstrate progressive worsening of the bilateral lung opacities and consolidations the identification of thickened and irregular pleural line and B lines in a variety of patterns $[9,10]$. We speculated that it could be useful also in monitoring hospitalized ventilated or intubated COVID-19 patients. However, in our experience, CXR and CT scan were performed rather than lung ultrasound in hospitalized patients to monitor lung involvement. Indeed, ultrasound has a limited sensitivity and specificity for the assessment of lung diseases because it has a limited capability in detecting pulmonary lesions that are deep and intrapulmonary and ultrasound findings of COVID-19 overlap with those from other viral pneumonia. CXR and $\mathrm{CT}$ are currently used in patients with suspected or confirmed COVID-19 pneumonia [2]. Baseline CXR and chest CT have a sensitivity of $69 \%$ and $97 \%$ for the diagnosis of COVID-19, respectively (Fig. 1) [11-13]. CT may allow to detect COVID-19 pneumonia even before real-time PCR $[12,13]$. However, the huge drawback of CT is its low specificity (i.e., 25-56\%) for COVID-19 pneumonia [12, 13], due to overlapping of imaging features with other viral or atypical pneumonia [14, 15].
Fig. 3 Typical imaging findings of COVID-19 pneumonia on chest $\mathrm{CT}$. a A 72-year-old man with COVID-19 infection: chest CT shows bilateral peripheral ground-glass opacities. b A 67 year-old man with COVID-19 infection: chest CT demonstrates extensive bilateral ground-glass opacities with subsegmental vessel enlargement (arrowheads) and traction bronchiectasis (arrows)

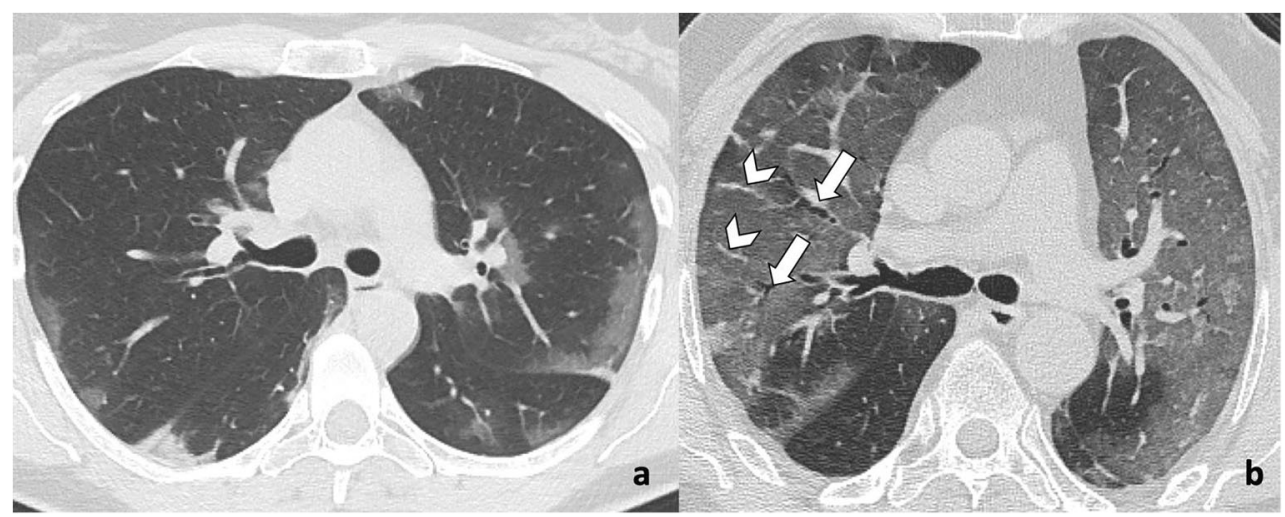


Fig. 4 Uncommon imaging findings of COVID-19 pneumonia on chest CT: halo sign and pleural effusion. a A 58-year-old man with COVID-19 infection: chest CT shows typical halo sign (arrow) characterized by a nodule surrounded by ground-glass opacity. b A 67-year-old woman with COVID-19 infection: chest $\mathrm{CT}$ demonstrates ground-glass opacities in the inferior lobes and mild bilateral pleural effusion

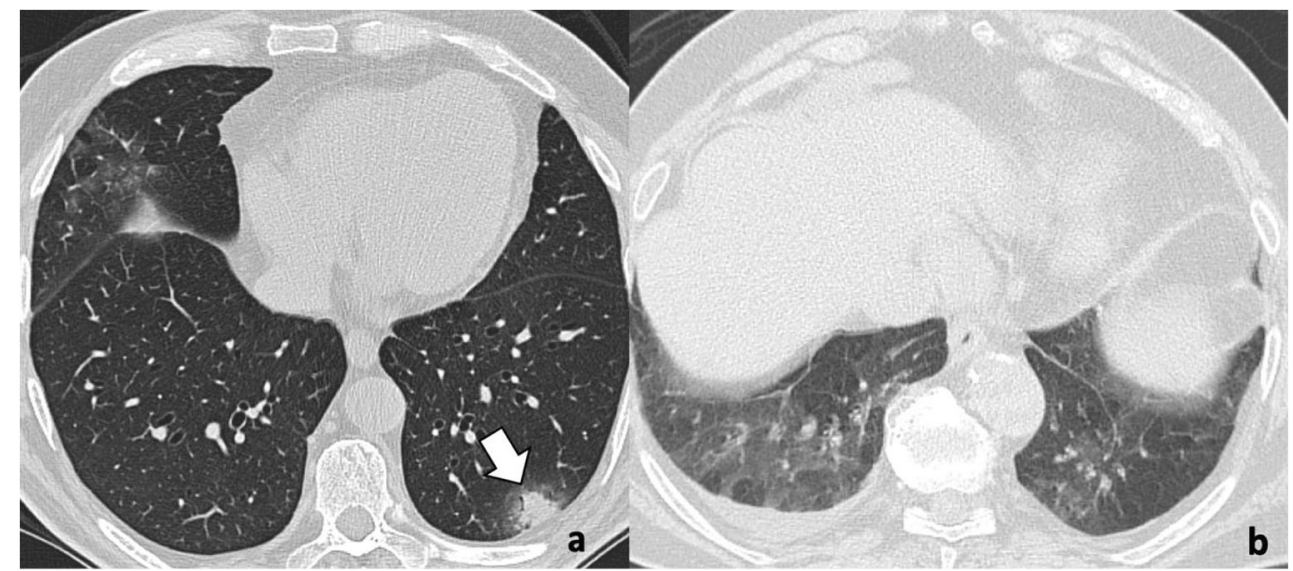

\section{Indications to imaging assessment}

The use of imaging in suspected cases of COVID-19 pneumonia is currently debated, with some authors highlighting the high sensitivity of CT $[12,13]$ and others showing that its routine use should be avoided given its low specificity [14, 15]. A multinational consensus statement from the Fleischner Society issued on April 2020 has recommended to avoid any imaging in suspected cases of COVID-19 and mild clinical symptoms unless there is any risk for disease progression and has indicated imaging only for medical triage of suspected cases of COVID-19 who present with moderate-severe clinical features and a high pre-test probability of disease [2]. In addition, imaging is indicated in patients with functional impairment after recovery to differentiate between expected abnormalities following COVID-19 infection and/or mechanical ventilation, versus a different and potentially treatable process [2].

Chest $\mathrm{CT}$ is indicated and widely adopted as first-line imaging technique in suspected cases of COVID-19 who present with moderate-severe clinical features and high pre-test probability of disease as well as in confirmed symptomatic cases $[2,16]$. Considering its high sensitivity, it is also questioned whether chest CT should be used as a screening tool in highly prevalent areas either as a stand-alone or as an adjunct to realtime PCR to exclude occult infection prior to surgery or intensive immunosuppressive therapies [2].

CXR is of little diagnostic value in early stages due to the low sensitivity (Fig. 1) and it is not indicated in the early course of the disease, except in resource-constrained environments with very high number of suspected COVID-19 symptomatic patients, long real-time PCR turnaround times, and lack of possibility to

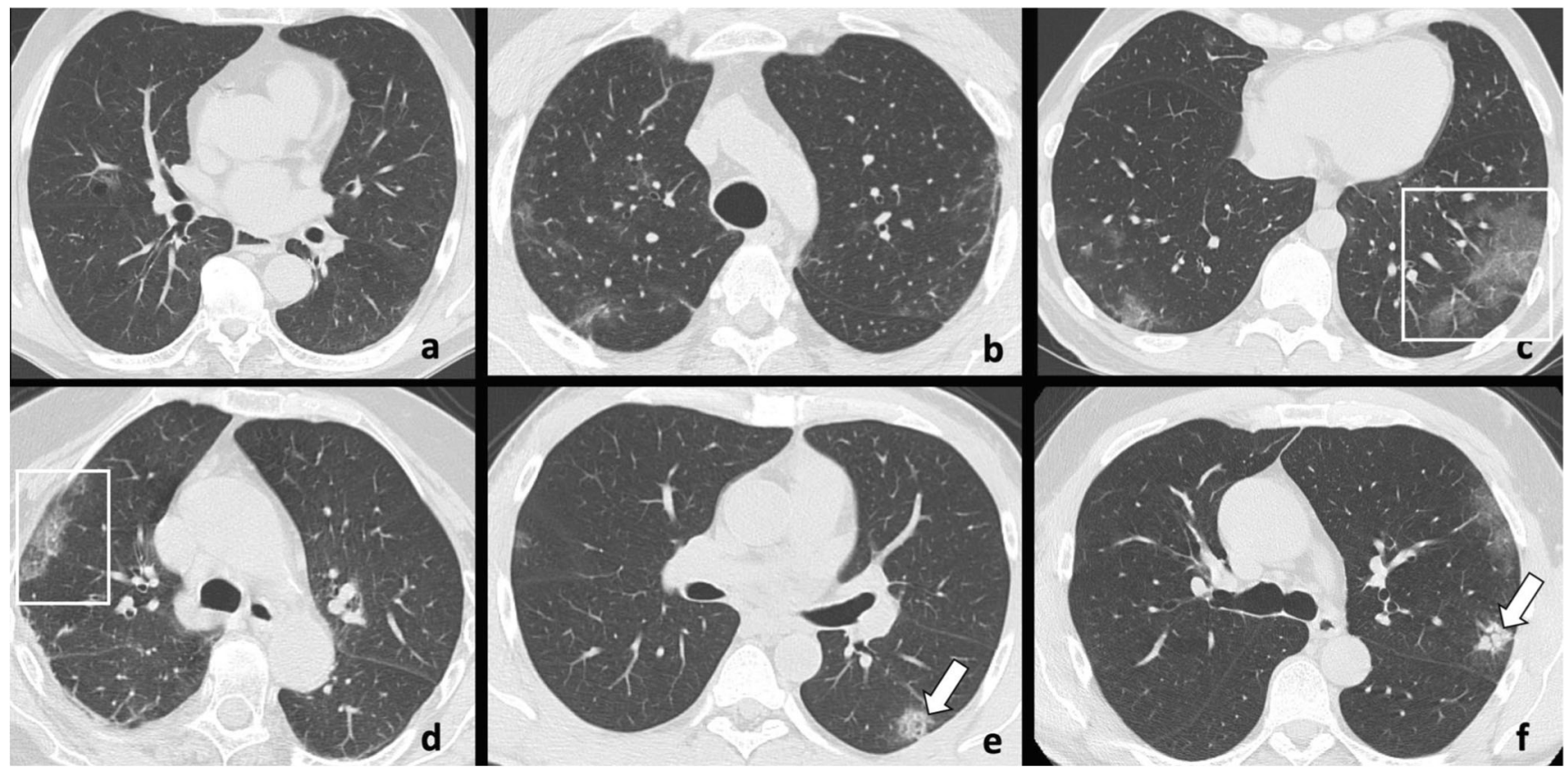

Fig. 5 Chest CT imaging findings in the ultra-early stage in six different patients with COVID-19 pneumonia range from single (a) or few (b, c, d) ground-glass opacities (frames) to focal small consolidations (e, f; arrows) 
Fig. 6 Chest CT imaging findings in the early stage of COVID-19 pneumonia. Chest CT in an 80year-old man with fever since 8 days (a, b) and in a 78-year-old $\operatorname{man}(\mathbf{c}, \mathbf{d})$ in the coronal $(\mathbf{a}, \mathbf{c})$ and axial $(\mathbf{b}, \mathbf{d})$ planes show extensive reticular pattern superimposed on the background of ground-glass opacity in the bilateral lungs, predominantly located in the upper lobes. Patient $1(\mathbf{a}, \mathbf{b})$ died after 3 days

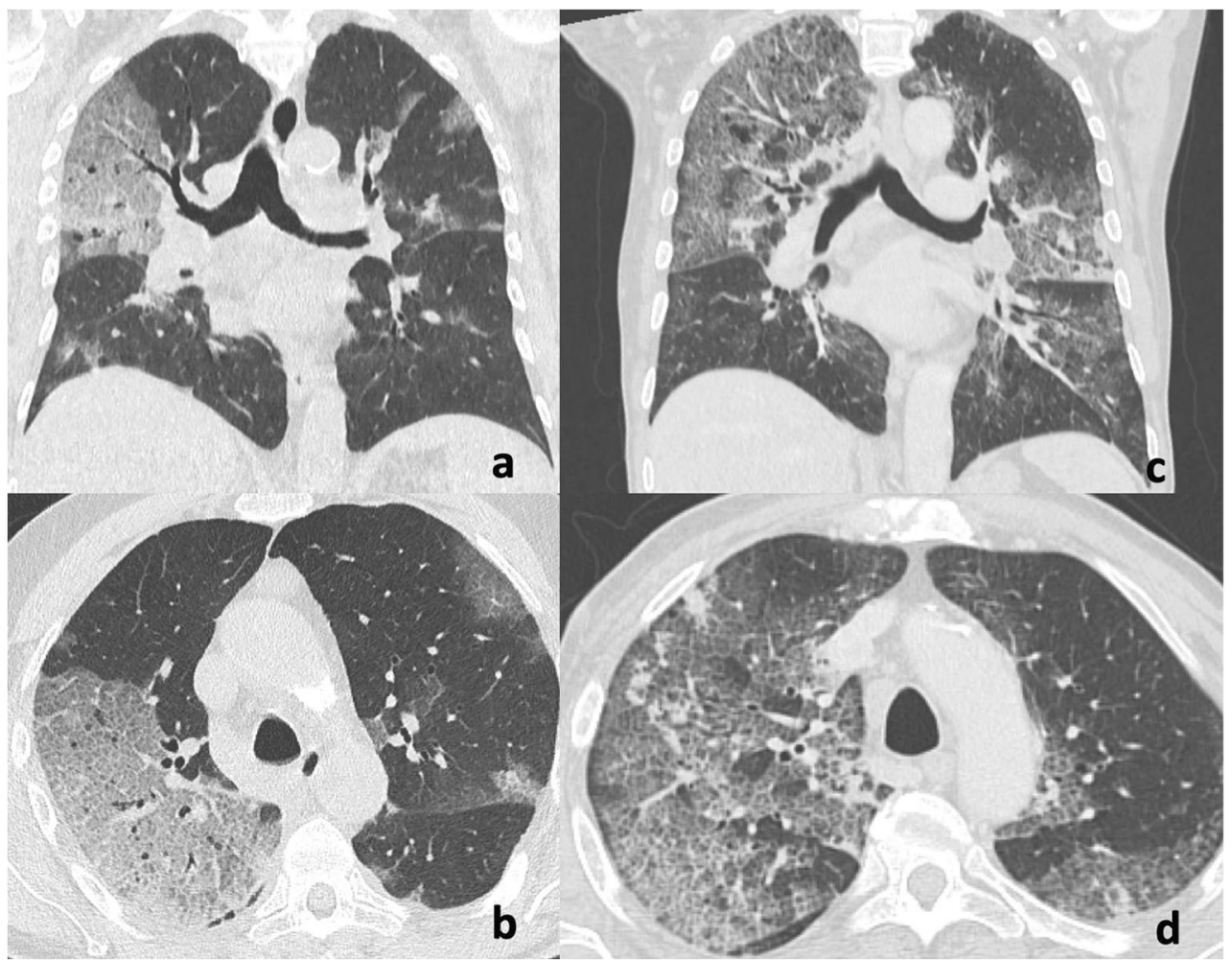

supersede chest radiographs by CT [2, 17]. CXR has a pivotal role in hospitalized patients for assessing disease progression, bacterial superinfection, pneumothorax, and pleural effusion [2]. In the intermediate to advanced stages of the disease, CXR may show progression of imaging features of acute respiratory distress syndrome in intensive care unit (ICU) patients (Fig. 2) and may help in guiding patient management [17]. However, CXR is not needed on a daily basis in stable intubated patients in ICU as there is no impact on important outcomes (mortality, length of stay, and ventilator days) as compared with on-demand CXR $[18,19]$.

\section{CXR and CT imaging findings}

The characteristic CT manifestations of COVID-19 pneumonia most commonly include bilateral and multilobar groundglass opacities (GGO) with peripheral distribution (Fig. 3a)
[17]. Interestingly, a common and typical finding of COVID-19 pneumonia is the presence of subsegmental vessel enlargement ( $>3 \mathrm{~mm}$ ) (Fig. 3b) and traction bronchiectasis within areas of GGO (Fig. 3b) [13, 20]. The "reversed halo" sign, pulmonary nodules with a halo sign (Fig. 4a) and pleural effusion (Fig. 4b) may be present but are uncommon CT features in COVID-19 [16].

CT findings of COVID-19 pneumonia vary with time [21] and different temporal stages have been described [15, 22] as follows:

- An ultra-early stage - i.e., asymptomatic patients with negative laboratory test but positive throat swab for 2019-nCoV within 1-2 weeks from COVID-19 infection-that demonstrates on CT single or few focal GGO, patchy consolidation, pulmonary nodules encircled by GGO, and air bronchograms (Fig. 5)
Fig. 7 Chest CT imaging findings in the rapid progression stage of COVID-19 pneumonia. Chest CT images in a 73-year-old man (a) and in a 59-year-old woman (b) show rapid progression from ground-glass opacities to consolidative opacities (frames), some with air bronchograms (b)
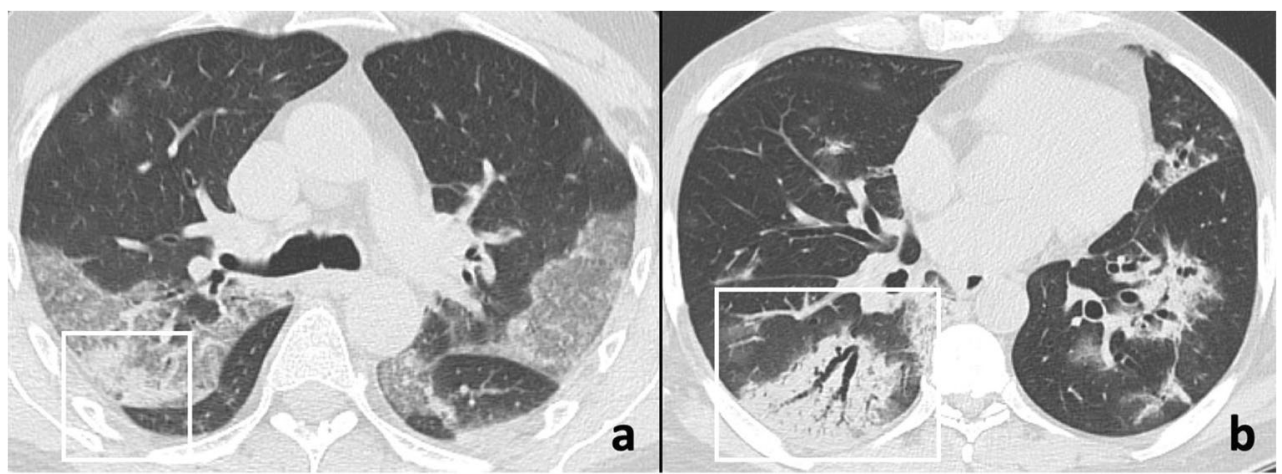


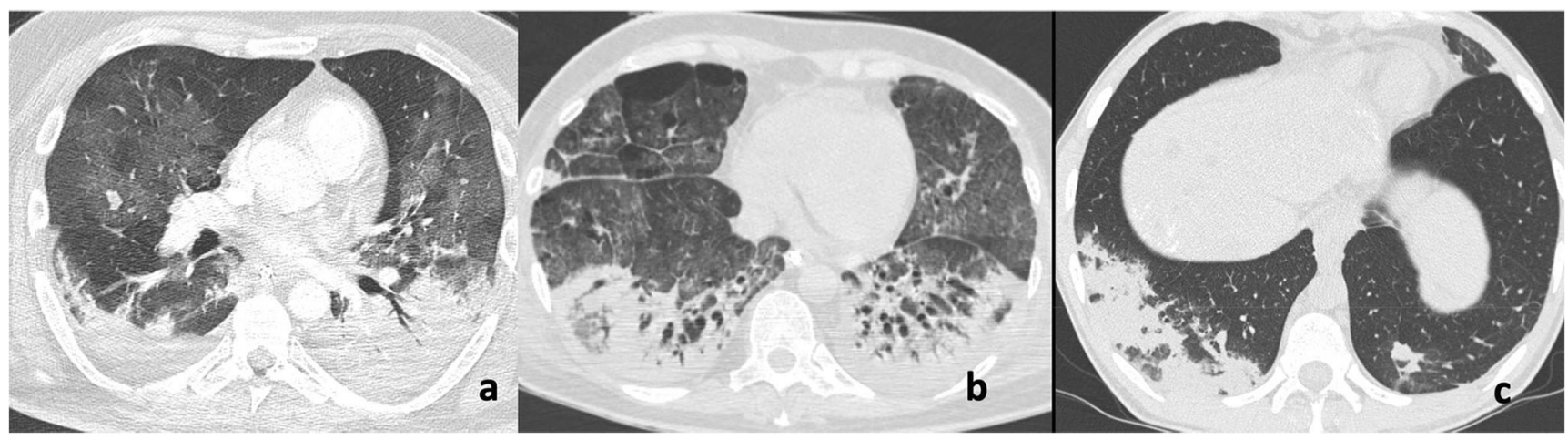

Fig. 8 Chest CT imaging findings in the consolidative stage of COVID-19 pneumonia in three different patients demonstrate multiple subpleural patchy consolidations in the bilateral lungs

- An early stage (1-3 days from onset of symptoms) when dilatation and congestion of alveolar septal capillary, exudation of fluid in alveolar cavity, and interlobular interstitial edema result into single or multiple scattered patchy or agglomerated GGO, separated by honeycomb-like or grid-like thickened of interlobular septa; of note, thickened interlobular and intralobular lines in combination with GGO is known as "crazy paving" pattern (Fig. 6)

- A rapid progression (3-7 days from onset of symptoms) when accumulation of a large number of cell-rich exudates in the alveoli, vascular expansion and interstitial exudation result into large, light consolidative opacities with air bronchograms (Fig. 7)

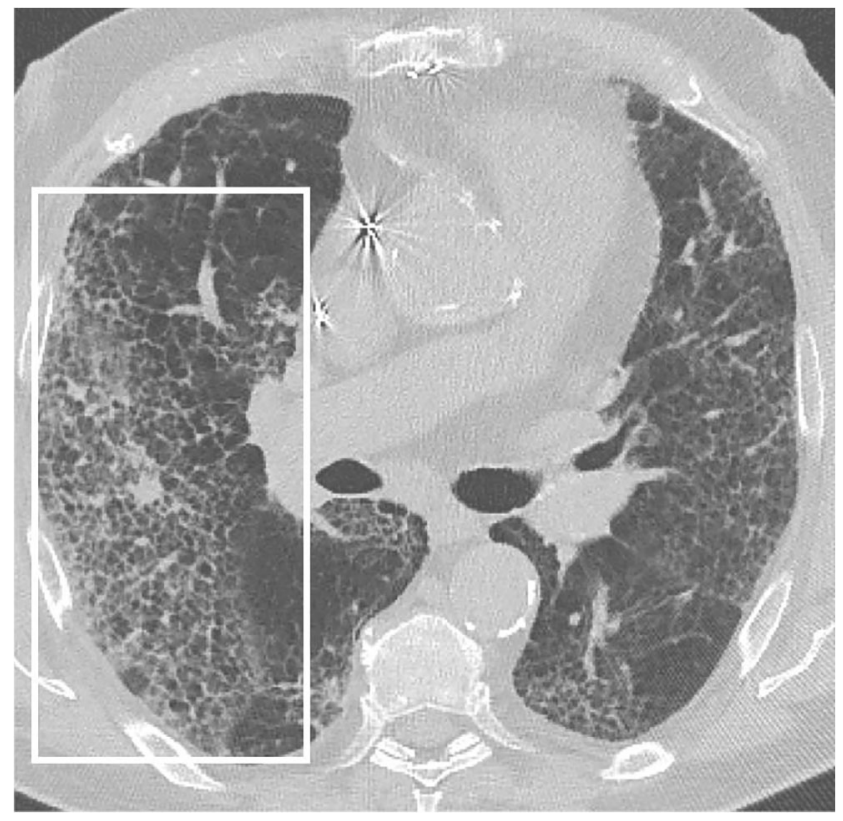

Fig. 9 A 78-year-old man with COVID-19 in dissipation stage. Axial chest CT shows interlobular septa thickening and reticular opacities (frame) after COVID-19 pneumonia
- A consolidation stage (7-14 days from onset of symptoms) when fibrous exudation of the alveolar cavity and disappearance of capillary congestion in the alveolar wall result into multiple patchy consolidations, and a progressive reduction in density and size of the consolidative opacities may be seen (Fig. 8)

- A dissipation stage (2-3 weeks from onset of symptoms) with grid-like thickening of interlobular septa, dispersed patchy consolidative opacities, reticular opacities, and bronchial wall thickening (Fig. 9)

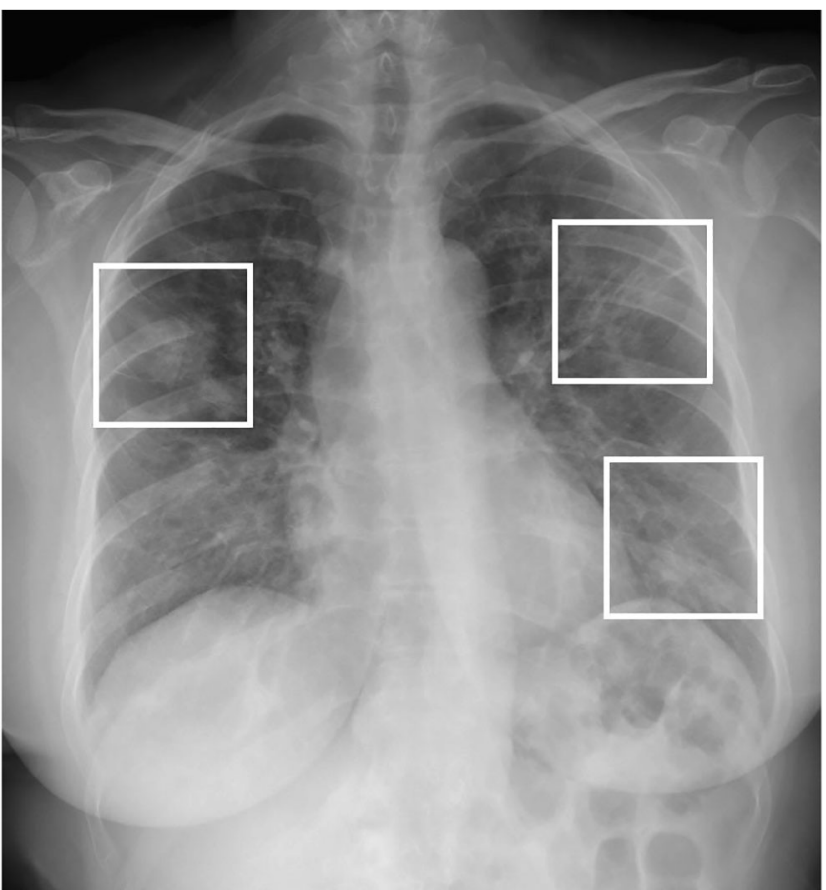

Fig. 10 Typical imaging findings of COVID-19 pneumonia on chest radiographs. A 58-year-old woman with COVID-19 infection: chest radiograph shows bilateral ground-glass opacities (frames) with predominant peripheral location 


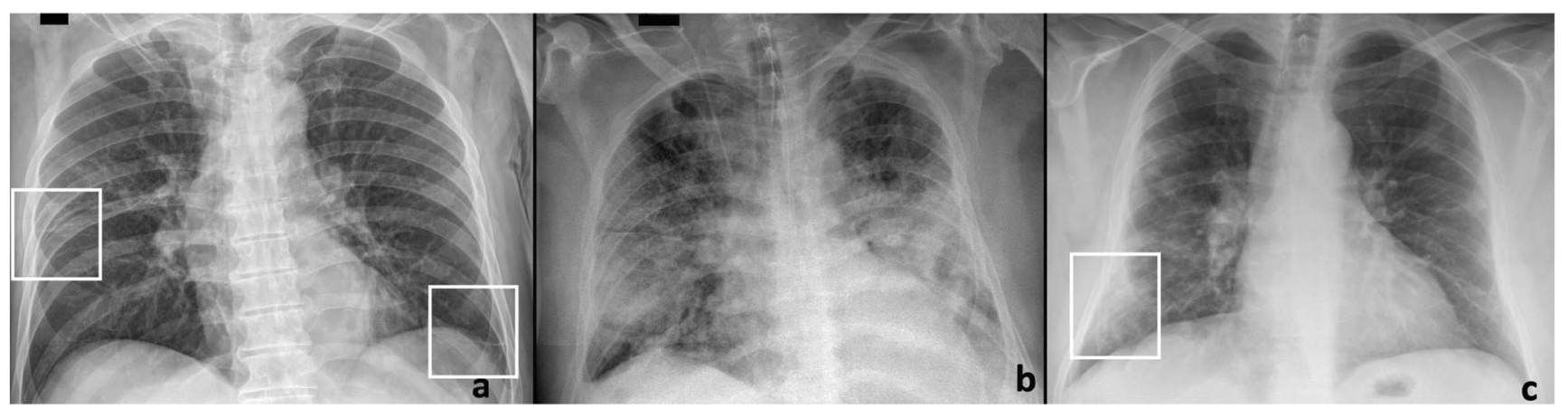

Fig. 11 Chest radiograph patterns of COVID-19 pneumonia. a An 84year-old man with hypotension and dyspnea since 7 days: CXR shows Kerley lines (frames) perpendicular to the pleura. b 68-year-old man with dyspnea and fever since 7 days and who died after 5 days: CXR shows

CXR shows similar findings of CT including bilateral pneumonia in about $63-73 \%$ of hospitalized patients, with interstitial infiltrates in $41-68 \%$ of the patients, peripheral distribution in $51 \%$ of the cases, with predominant involvement of the lower lung zones in $63 \%$ (Fig. 10) [11, 23]. CXR findings in COVID-19 patients may present with an interstitial pattern (Fig. 11a) with reticular or reticulonodular hypodiaphany and oftentimes Kerley lines that are perpendicular to the pleura and indicate interstitial thickening, a consolidative pattern with

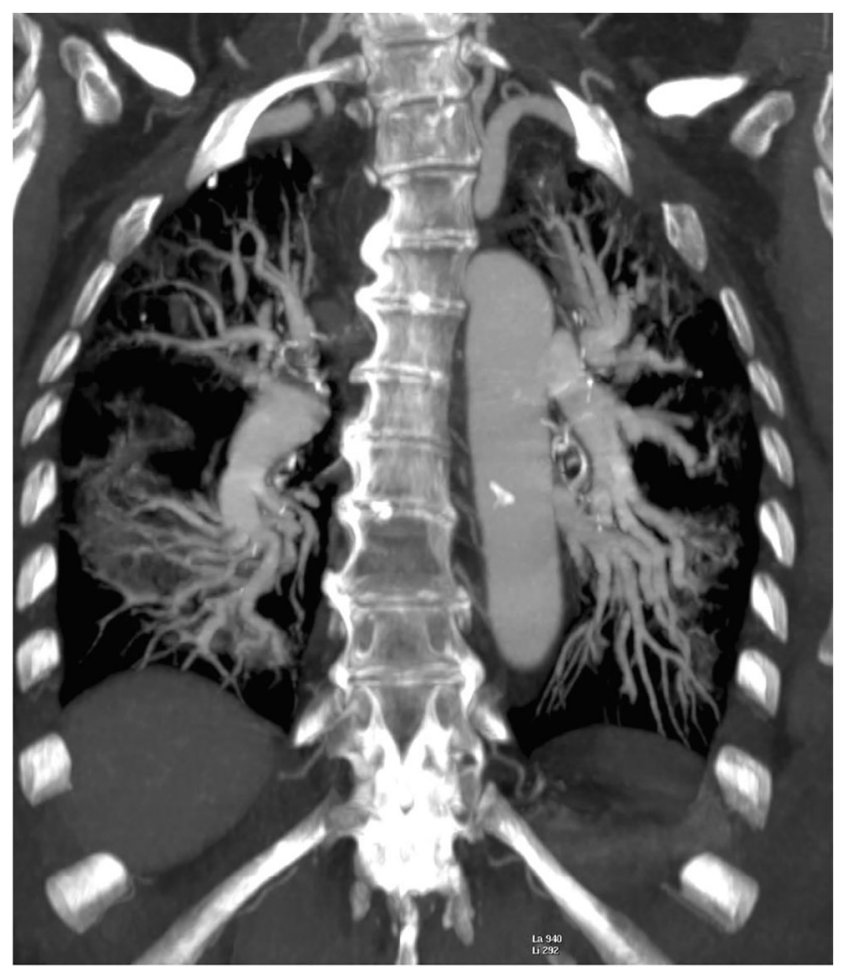

Fig. 12 An 83-year-old woman with COVID-19 pneumonia. Chest CT angiography reconstructed using maximum intensity projection (MIP) demonstrates enlargement of pulmonary segmental vessel and peripheral ground-glass opacities consolidative pattern and ground-glass opacities. c A 55-year-old man with fever and dyspnea since 4 days: CXR shows combination of the reticular and consolidative patterns (frame)

ill-defined hypodiaphania including ground-glass opacification (Fig. 11b) - a term usually used on CT to describe to an area of increased attenuation with preserved bronchial and vascular markings - or homogeneous opacification with air bronchogram, or a combination of the reticular and consolidative patterns (Fig. 11c).

The severity and extent of the disease during hospitalization may be assessed using a CXR severity score obtained by adapting and simplifying the radiographic assessment of lung edema (RALE) score proposed by Warren et al. [24] and modified by Wong et al. [11]. According to this CXR severity score, a score of $0-4$ is assigned to each lung depending on the extent of involvement by consolidation or ground-glass opacities $(0$ : no involvement; 1 : $<25 \%$; $2: 25-50 \% ; 3: 50-75 \%$; 4 : $>75 \%$ involvement), and then the scores for each lung is summed to produce the final severity score [11]. The CXR severity score is not routinely used in the majority of radiology departments, but it is the authors' opinion that if this score is included in radiology reports, it could potentially simplify COVID-19 disease monitoring for clinicians. The peak severity according to CXR severity score in COVID-19 patients is reached at 10-12 days [11].

\section{Imaging for patient management}

The description of CT pattern may have important implications for patient management. To date, about $3 \%$ of currently infected patients has serious or critical conditions [25], and about $17 \%$ of hospitalized patients needs ICU admission $[26,27]$. Radiologists may be helpful in identifying patients at higher risk of lung disease worsening. Indeed, the presence of tubular size increase of pulmonary segmental vessel around and within the lesions with normally ventilated adjacent lung parenchyma (Fig. 12) has been suggested as an early alert radiological sign to predict initial lung deterioration [28]. Indeed, some authors believe that the presence of enlarged pulmonary vessels may indicate an inflammatory cell infiltration of the vessels [29] or might be due to pro-inflammatory 


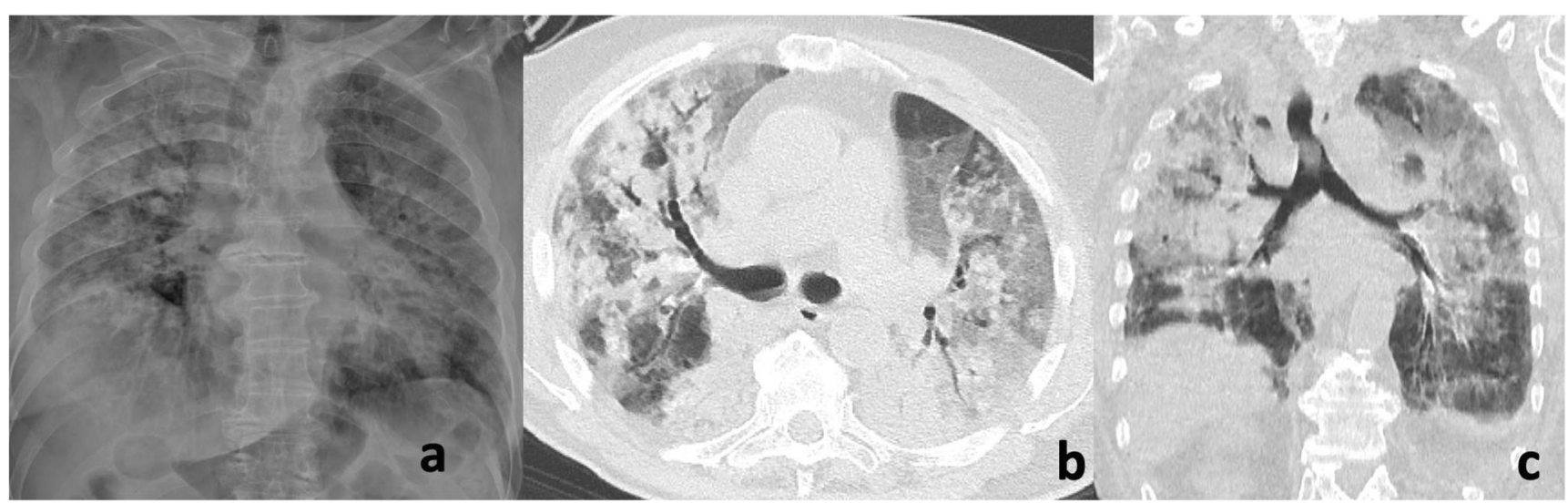

Fig. 13 An 85-year-old woman with worsening dyspnea since 7 days, history of arterial hypertension, lymphopenia, and with COVID-19 pneumonia progressed to acute respiratory distress syndrome. Chest radiograph (a), axial (b), and coronal (c) CT images show extensive ground-glass opacities alternating with consolidations in both lungs. The patient died 2 days after CT factors [30], thus potentially indicating that the patient is in the second stage of COVID-19 lung involvement.

Chest CT may help in guiding therapeutic strategy. CXR and CT may demonstrate findings of acute respiratory distress syndrome (ARDS) (Fig. 13) including bilateral opacities, not fully explained by volume overload, lobar or lung collapse, or nodules [31]. The most common CT finding of ARDS is the presence of large lung infiltrates with loss of ventilation, especially in the posterior and basal regions, with ground-glass opacities alternating with consolidations [32]. However, imaging findings of ARDS vary depending on the stage of the disease ranging from an exudative acute phase in the first week characterized by bilateral and symmetrical air space

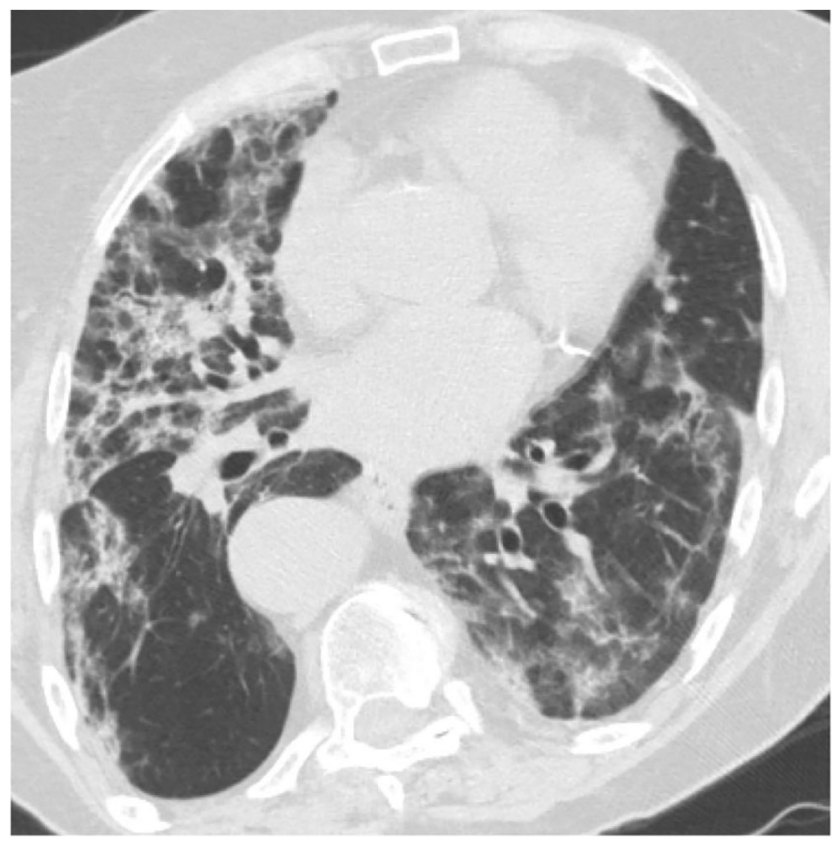

Fig. 14 An 85-year-old woman with fever, cough, and dyspnea images after 2 weeks of COVID-19 infection. Axial chest CT shows coarse reticular pattern with areas of fine fibrosis in both lungs and interstitial opacities on CXR, and opacifications showing an antero-posterior density gradient characterized by dense bilateral consolidation in the dependent lung and a background of widespread GGO or normal lung in the nondepended portions of the lung, to a proliferative intermediate phase in the second week with overall stability of the imaging findings and a fibrotic late phase after 2 weeks with normal or reticular opacities on CXR, and eventually a coarse reticular pattern with areas of fine fibrosis on CT (Fig. 14) [32]. It is important to identify patients with COVID-19 pneumonia progressing to ARDS because these patients could benefit from corticosteroid treatment according to Xu et al. [6]. In addition, in patients with severe ARDS, prone ventilation for 12-16 h per day is indicated by World Health Organization Interim guidance because it improves oxygenation and respiratory compliance compared with supine positioning [33, 34]. Chest imaging is also considered important in World Health Organization Interim guidance V 1.2 when findings of pulmonary edema are evident because, in these cases, it is indicated to reduce or discontinue fluid administration to avoid volume overload [33].

\section{Conclusions}

To conclude, imaging appearance of COVID-19 pneumonia may vary depending on the stage of the disease. CT is indicated for medical triage of suspected cases of COVID-19 who present with moderate-severe clinical features and a high pretest probability of disease and may be useful in hospitalized patients to identify signs that indicate potential lung disease worsening. Chest radiographs are more commonly used for assessing disease progression, bacterial superinfection, pneumothorax, and pleural effusion. A comprehensive knowledge of the typical findings of COVID-19 pneumonia in the different stages is of utmost importance because some of the 
imaging findings have a translational importance in clinical practice for patient therapy.

\section{Compliance with ethical standards}

Conflict of interest The authors declare that they have no conflict of interests.

\section{References}

1. https://www.who.int/emergencies/diseases/novel-coronavirus2019/situation-reports/.Accessed on April 20, 2020

2. Rubin GD, Ryerson CJ, Haramati LB et al (2020) The role of chest imaging in patient management during the COVID-19 pandemic: a multinational consensus statement from the Fleischner Society. Radiology 201365

3. Wan Y, Shang J, Graham R, Baric RS, Li F (2020) Receptor recognition by the novel coronavirus from Wuhan: an analysis based on decade-long structural studies of SARS coronavirus. J Virol 94: e00127-e00120

4. Xu H, Zhong L, Deng J, Peng J, Dan H, Zeng X, Li T, Chen Q (2020) High expression of ACE2 receptor of 2019-nCoV on the epithelial cells of oral mucosa. Int J Oral Sci 12:8

5. Hoffmann M, Kleine-Weber H, Schroeder S et al (2020) SARSCoV-2 cell entry depends on ACE2 and TMPRSS2 and is blocked by a clinically proven protease inhibitor. Cell 181:271-280.e8

6. Xu Z, Shi L, Wang Y et al (2020) Pathological findings of COVID19 associated with acute respiratory distress syndrome. Lancet Respir Med 8:420-422 published correction appears in Lancet Respir Med. 2020 Feb 25;:

7. Tian S, Hu W, Niu L, Liu H, Xu H, Xiao SY (2020) Pulmonary pathology of early-phase 2019 novel coronavirus (COVID-19) pneumonia in two patients with lung cancer. J Thorac Oncol 15(5):700-704 published online ahead of print, $2020 \mathrm{Feb} 28$. https://doi.org/10.1016/j.jtho.2020.02.010

8. Shi Y, Wang Y, Shao C, Huang J, Gan J, Huang X, Bucci E, Piacentini M, Ippolito G, Melino G (2020) COVID-19 infection: the perspectives on immune responses published online ahead of print, 2020 Mar 23. Cell Death Differ 27:1451-1454. https://doi. org/10.1038/s41418-020-0530-3

9. Peng QY, Wang XT, Zhang LN; Chinese Critical Care Ultrasound Study Group (CCUSG) 2020. Findings of lung ultrasonography of novel corona virus pneumonia during the 2019-2020 epidemic published online ahead of print, 2020 Mar 12. Intensive Care Med;1-2

10. Soldati G, Smargiassi A, Inchingolo R et al (2020) Is there a role for lung ultrasound during the COVID-19 pandemic? published online ahead of print, 2020 Mar 20. J Ultrasound Med 39(7):1459-1462. https://doi.org/10.1002/jum.15284

11. Wong HYF, Lam HYS, Fong AH, et al. 2019 Frequency and distribution of chest radiographic findings in COVID-19 positive patients published online ahead of print, 2019 Mar 27. Radiology ;201160

12. Ai T, Yang Z, Hou H et al (2020) Correlation of chest CT and RTPCR testing in coronavirus disease 2019 (COVID-19) in China: a report of 1014 cases [published online ahead of print, $2020 \mathrm{Feb} 26$ ]. Radiology 26:200642

13. Caruso D, Zerunian M, Polici M et al (2020) Chest CT features of COVID-19 in Rome, Italy [published online ahead of print, 2020 Apr 3]. Radiology 3:201237

14. Hosseiny M, Kooraki S, Gholamrezanezhad A, Reddy S, Myers L (2020) Radiology perspective of coronavirus disease 2019
(COVID-19): lessons from severe acute respiratory syndrome and Middle East respiratory syndrome [published online ahead of print, 2020 Feb 28]. AJR Am J Roentgenol 214:1-5

15. Jin YH, Cai L, Cheng ZS et al (2020) A rapid advice guideline for the diagnosis and treatment of 2019 novel coronavirus (2019nCoV) infected pneumonia (standard version). Mil Med Res 7:4

16. Li Y, Xia L 2020. Coronavirus disease 2019 (COVID-19): role of chest $\mathrm{CT}$ in diagnosis and management [published online ahead of print, 2020 Mar 4]. AJR Am J Roentgenol ;1-7

17. Salehi S, Abedi A, Balakrishnan S, Gholamrezanezhad A (2020) Coronavirus disease 2019 (COVID-19): a systematic review of imaging findings in 919 patients [published online ahead of print, 2020 Mar 14]. AJR Am J Roentgenol 215:1-7

18. Oba Y, Zaza T (2010) Abandoning daily routine chest radiography in the intensive care unit: meta-analysis. Radiology 255(2):386395. https://doi.org/10.1148/radiol.10090946

19. Hejblum G, Chalumeau-Lemoine L, Ioos V, Boëlle PY, Salomon L, Simon T, Vibert JF, Guidet B (2009) Comparison of routine and on-demand prescription of chest radiographs in mechanically ventilated adults: a multicentre, cluster-randomised, two-period crossover study. Lancet 374:1687-1693

20. Zhao W, Zhong Z, Xie X, Yu Q, Liu J (2020) Relation between chest $\mathrm{CT}$ findings and clinical conditions of coronavirus disease (COVID-19) pneumonia: a multicenter study [published online ahead of print, 2020 Mar 3. AJR Am J Roentgenol 214(5):1-6

21. Bernheim A, Mei X, Huang M, et al. 2020 Chest CT findings in coronavirus disease-19 (COVID-19): relationship to duration of infection [published online ahead of print, $2020 \mathrm{Feb} 20$ ]. Radiology;200463

22. Pan F, Ye T, Sun P et al (2020) Time Course of Lung Changes On Chest CT During Recovery From 2019 Novel coronavirus (COVID-19) pneumonia. Radiology 295(3):715-721 published online ahead of print, $2020 \mathrm{Feb} 13$

23. Rodriguez-Morales AJ, Cardona-Ospina JA, Gutiérrez-Ocampo E et al (2020) Clinical, laboratory and imaging features of COVID19: a systematic review and meta-analysis. Travel Med Infect Dis 34:101623 published online ahead of print, 2020 Mar 13

24. Warren MA, Zhao Z, Koyama T, Bastarache JA, Shaver CM, Semler MW, Rice TW, Matthay MA, Calfee CS, Ware LB (2018) Severity scoring of lung oedema on the chest radiograph is associated with clinical outcomes in ARDS. Thorax 73:840-846

25. https://www.worldometers.info/coronavirus/\#countries Accessed April 19, 2020

26. https://www.cdc.gov/mmwr/volumes/69/wr/mm6913e2.htm\#F1_ down Accessed April 19, 2020

27. Grasselli G, Pesenti A, Cecconi M (2020) Critical care utilization for the COVID-19 outbreak in Lombardy, Italy: early experience and forecast during an emergency response [published online ahead of print, 2020 mar 13]. JAMA 323:1545. https://doi.org/10.1001/ jama.2020.4031

28. Albarello F, Pianura E, Di Stefano F et al (2020) 2019-novel coronavirus severe adult respiratory distress syndrome in two cases in Italy: an uncommon radiological presentation [published online ahead of print, 2020 Feb 26]. Int J Infect Dis 93:192-197

29. Han X, Cao Y, Jiang N, et al. 2020 Novel coronavirus pneumonia (COVID-19) progression course in 17 discharged patients: comparison of clinical and thin-section CT features during recovery [published online ahead of print, 2020 Mar 30]. Clin Infect Dis. ;ciaa271

30. Ye Z, Zhang Y, Wang Y, Huang Z, Song B 2020 Chest CT manifestations of new coronavirus disease 2019 (COVID-19): a pictorial review. Eur Radiol. doi: https://doi.org/10.1007/s00330-02006801-0

31. Bernard GR, Artigas A, Brigham KL, Carlet J, Falke K, Hudson L, Lamy M, Legall JR, Morris A, Spragg R (1994) The AmericanEuropean Consensus Conference on ARDS. Definitions, 
mechanisms, relevant outcomes, and clinical trial coordination. Am J Respir Crit Care Med 149:818-824

32. Sheard S, Rao P, Devaraj A (2012) Imaging of acute respiratory distress syndrome. Respir Care 57:607-612

33. World Health Organizazion Clinical management of severe acute respiratory infection (SARI) when COVID-19 disease is suspected. Interim guidance V 1.2. 13 March 2020, Accessed Apr 19, 2020
34. Pelosi P, Brazzi L, Gattinoni L (2002) Prone position in acute respiratory distress syndrome. Eur Respir J 20:1017-1028

Publisher's note Springer Nature remains neutral with regard to jurisdictional claims in published maps and institutional affiliations. 